\title{
Improvements in the Advanced Navy Aerosol Model (ANAM)
}

\author{
A.M.J. van Eijk ${ }^{* a}$ and D.L. Merritt ${ }^{b}$ \\ ${ }^{a}$ TNO Defence, Security and Safety, The Hague, The Netherlands, \\ b.D. Fontana \& Associates, Inc, Ramona CA, USA
}

\begin{abstract}
The Navy Aerosol Model (NAM) is widely used as an engineering tool to provide a quick estimate of the aerosol extinction in the marine environment. Since its introduction, several shortcomings of NAM have been identified that are being addressed by the development of the Advanced Navy Aerosol Model (ANAM). At present, the Advanced Navy Aerosol Model has been reviewed as concerns its production mode. The two separate production modes $\left(3^{\text {rd }}\right.$ and $4^{\text {th }}$ modes in ANAM4) have been replaced by a single production mode in ANAM5. The shape of the new production mode is given by two sea spray source functions taken from literature, Vignati et al. and Smith and Harrison. The intensity of the new production mode in ANAM5 at a particular height above the surface is governed by a transfer function that depends on radius and wind speed. The production mode in ANAM5 has several tuning parameters that have been optimized by comparing ANAM5 concentration predictions to experimental aerosol data. ANAM5 performs better than ANAM4 in predicting the concentrations of large aerosols in open ocean conditions, but the performance is reduced in the coastal zone. This may be due to the presence of a strong advection mode that is currently not well taken into account by the ANAM.
\end{abstract}

Keywords: marine aerosol, sea spray production, ANAM, empirical modeling

\section{INTRODUCTION}

Electro-optical sensors are presently used for a wide range of applications in the marine environment, e.g., for surveillance, ranging, and classification. The performance of state-of-the-art sensors is generally not determined by system design and technological limitations, but rather by the intervening atmosphere. Propagation of electro-optical radiation through the atmosphere can be severely limited by absorption and scattering of radiation (transmission losses). Turbulence and refraction may cause severe image distortion, thereby hampering the recognition of targets. Since these adverse effects are strongly dependent on the environmental conditions, there is a need for tools that can assess the sensor performance as a function of the meteorological scenario.

Absorption and scattering are caused by the presence of molecules and aerosols, which necessitates that the atmospheric composition and concentration of these constituents are known. The molecular part is relatively constant (apart from water vapor) and the scattering and absorption characteristics of the various molecular species are well-known. Hence, reliable codes, such as the USAF MODTRAN ${ }^{1}$ code are available to assess the molecular component. The composition and concentration of the atmospheric aerosols is much more variable, and considerable effort has been spent on the development of aerosol transmission codes. To predict aerosol transmission losses along a propagation path, the model must estimate the concentration and composition (refractive index) of the various aerosol species. Subsequently, Mie theory ${ }^{2}$ provides the absorption and scattering coefficients.

The open ocean environment provided a relatively simple case for an aerosol transmission model. Here, the problem is less strenuous because the majority of the aerosol consists of sea spray, generated at the wave surface, and homogeneous conditions exist over appreciable spatial domains. The sea spray component in the marine atmospheric surface layer (MASL, up to several tens of meters) could be successfully described by the Navy Aerosol Model (NAM), developed by Stu Gathman. ${ }^{3}$ This parametric model applies statistical relations between (surface) meteorological parameters and the aerosol concentration, and thereby provides a general estimate of the aerosol concentration for the MASL. Although

\footnotetext{
* Contact information: lex.vaneijk@tno.nl, phone: +31 70374 0451, TNO Defence, Security and Safety, P.O. Box 96864, 2509 JG The Hague, The Netherlands; http://www.tno.nl
}

Atmospheric Optical Modeling, Measurement, and Simulation II, edited by Stephen M. Hammel, Anton Kohnle, Proc. of SPIE Vol. 6303, 63030M, (2006) · 0277-786X/06/\$15 · doi: 10.1117/12.680295 
parametric, the NAM relates the aerosol concentration to key processes such as production, evaporation and advection, which is reflected in the set of input meteorological parameters. The applicability of the NAM was later extended throughout the boundary layer by the Navy Oceanic Vertical Aerosol Model (NOVAM). ${ }^{4}$ This approach combined aerosol vertical dispersion algorithms with meteorological boundary layer parameterizations, which necessitated additional input parameters (radiosonde observations). The combined NAM-NOVAM package is currently available in the USAF MODTRAN ${ }^{1}$ code.

Over the years, the Navy Aerosol Model (NAM) has been widely used to assess the aerosol extinction. The popularity of the model results from its availability through MODTRAN, but also from its relatively small set of input parameters and its minimal computational requirements. It is generally acknowledged that the NAM provides an adequate engineering tool to estimate the aerosol extinction for open ocean conditions. ${ }^{5}$

However, the NAM has also received criticism over the years, especially as concerns the reduced performance in coastal regions ${ }^{6}$ and the difficulty in specifying a proper value in the littoral zone for one of the input parameters, i.e., the air mass parameter. Furthermore, experimental evidence ${ }^{7}$ suggested that the NAM underestimated the concentration of larger aerosols and therefore underestimated the extinction, ${ }^{8}$ especially in the 8-12 $\mu \mathrm{m}$ wavelength domain. Finally, some of the NAM parameters and equations have been questioned, e.g., the center diameters of particular modes ${ }^{5}$ (see also section 2) and the relative humidity dependence.

Since the conception of the NAM, elaborate numerical models ${ }^{10,11}$ have been developed that assess the aerosol life-cycle in more detail as concerns the larger aerosols near the surface behavior or the larger advection processes. In addition, satellite-based sensors have greatly enhanced our capabilities of assessing the aerosol concentration and composition in the coastal environment. However, the application of these new tools is not always straightforward since data access may be limited, computational demands can be high and trained operators are generally required. Thus, there is still a need for a relatively simple tool that can provide a first order estimate for the aerosol extinction. This provides the impetus for our efforts to continue the development of the NAM and to remedy its known deficiencies. The improved NAM has been named the Advanced Navy Aerosol Model (ANAM).

This paper addresses one aspect of the NAM improvements, i.e., the production of marine aerosols. The next section provides a more detailed description of the NAM and a report of the initial ANAM development, which focused on the underestimation of the contribution of larger marine aerosols in the original NAM. Subsequently, the new production mode in the ANAM is presented and an initial report on the tuning process of this new mode is given.

\section{NAM OVERVIEW AND INITIAL ANAM DEVELOPMENT}

The basic $\mathrm{NAM}^{3}$ was presented by Gathman in 1983, and represented one of the first efforts to model aerosol extinction solely on the basis of meteorological parameters. The NAM applies a two-step procedure to provide the aerosol extinction at ship's deck height for open ocean conditions. In the first step, the aerosol concentration is calculated using statistical relations between the concentration and meteorological parameters. This step is followed by a Mie calculation ${ }^{2}$ to yield aerosol extinction (and scattering). The original NAM has been updated on the basis of new experimental ${ }^{7,12}$ and theoretical ${ }^{13,14}$ evidence, and is presently included in the widely used USAF MODTRAN transmission code. ${ }^{1}$

The basic NAM describes the aerosol size distribution by a superposition of three lognormal curves ('modes'), cf. figure 1a (next page). Each mode is characterised by a width (assumed constant), a centre radius and amplitude. The centre radii of the modes are nominally $0.03,0.24$ and $2.0 \mu \mathrm{m}$, but are adjusted as function of the relative humidity. The largest or third mode $(2 \mu \mathrm{m})$ consists of freshly produced marine aerosols. Its amplitude is determined by the instantaneous wind speed. The second mode $(0.24 \mu \mathrm{m})$ consists of marine aerosols that have spent some time in the atmosphere ("aged" marine mode) and have adjusted their size to the ambient conditions. Since these particles have been produced elsewhere and transported to their present location, the amplitude of the second mode is determined by the wind speed history. Finally, the first mode $(0.03 \mu \mathrm{m})$ consists of fine particles that constitute a marine background concentration. These particles have been aloft for such a long time that there presence is no longer governed by the instantaneous wind speed or even the wind speed history. To control the amplitude of the $1^{\text {st }}$ mode, Gathman introduced the air mass parameter (AMP). Unfortunately, the value of the AMP was not defined on the basis of meteorological parameters, but rather by an educated guess of the operator. 

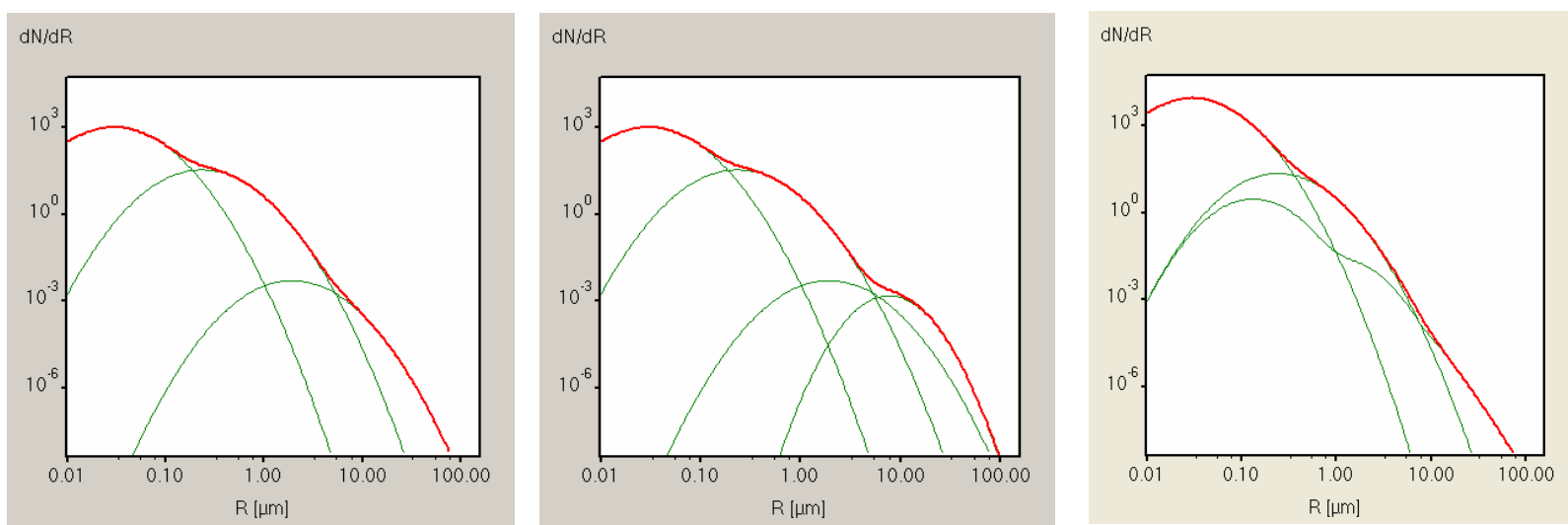

Figure 1a (left) NAM distribution plot; Figure 1b (middle) ANAM4 distribution plot; Figure 1c (right) ANAM5 distribution plot showing the new production mode

The 3-mode model described above constitutes a pure sea-spray aerosol model. However, an atmosphere containing only sea-spray aerosols is rare and limited to the most remote oceanic environments. Therefore, Gathman included a $0^{\text {th }}$ mode in the NAM, which represented a contribution by dust particles. The centre radius of the $0^{\text {th }}$ mode was placed at $0.03 \mu \mathrm{m}$ (just like the $1^{\text {st }}$ mode), but in contrast to the sea salt particles of the $1^{\text {st }}$ mode the dust particles were considered to be non-hygroscopic (no dependence on relative humidity). The role of the air mass parameter was extended to control the total amplitude of the $0^{\text {th }}$ and $1^{\text {st }}$ modes together. Furthermore, the AMP controlled the ratio between the $0^{\text {th }}$ and $1^{\text {st }}$ modes by a step-function: the $0^{\text {th }}$ mode was absent for $\mathrm{AMP}<5$ and contributed $30 \%$ to the total concentration for $\mathrm{AMP}>5$.

Before attempting to apply NAM in the littoral environment, it should be realized that the aerosol in coastal zone consists of a complex mixture of three types of aerosols. The first type is generated at the sea surface by the interaction between wind and waves (marine aerosols); the second type is generated at the land-sea interface (surf aerosols); the third type is generated over-land by natural and/or anthropogenic sources ("continental" aerosols). A further complication is that the continental aerosols themselves constitute a mixture: they vary considerably in chemical composition and consequently, refractive index. The exact concentration and composition of the aerosol mixture depends on a multitude of (meteorological) parameters, such as wind speed and wind direction, and the distance to the various sources. Due to the relatively rapid changes in meteorological conditions in the coastal zone, and due to the fact that many over-land sources are highly localized, the aerosol mixture is spatially and temporally inhomogeneous.

The previous paragraph explains the reduced performance of the NAM in the coastal zone: the model offers just two aerosol species (marine and dust), and just one parameter (AMP) that controls amplitude and ratio of the two modes. As mentioned in the introduction, this paper does not explicitly address the upgrades to NAM for the coastal zone. However, since the release of the basic NAM, efforts have been reported that suggest the need to include more aerosol species ${ }^{15}$ and to replace the AMP by fetch. ${ }^{16,17}$ The fetch represents the distance an air mass has traveled over water, and thereby provides a first indication for the ratio of marine versus continental aerosols. The longer the air mass has spent over water, the more continental particles will have dispersed and deposited, and the more marine particles have been generated.

It should be realized that Gathman based the NAM on experimental data that was mainly collected in the 1970s. Not only was the available data limited, the equipment of that time was also not very sensitive to the collection of very large particles (radius in excess of 10 microns). This became apparent at the end of the 1980s when DeLeeuw applied the Rotorod $^{*}$ technique to asses the near-surface concentrations of giant marine aerosols. It was found that NAM

\footnotetext{
* The simple Rotorod technique ${ }^{18-20}$, an active impactor sampling method that was first successfully applied over a marine surface by DeLeeuw ${ }^{7,21-24}$, has allowed the collection of one of the largest sets of near-surface large aerosol data. Two reports ${ }^{25,26}$ provide an extensive discussion of the Rotorod technique and its reliability.
} 
underestimated the concentration of these large particles. This led to the development of the ANAM4 model in the late 1990s.

The Advanced Navy Aerosol model (ANAM) adds a $4^{\text {th }}$ lognormal mode to NAM, centred at a nominal radius of 8 microns, to account for the largest marine particles. The $4^{\text {th }}$ mode parameters have been found from a regression analysis of Rotorod data and meteorological parameters. The initial analysis ${ }^{27}$ showed that the amplitude of the $4^{\text {th }}$ mode should be a function of the instantaneous wind speed (production of marine aerosols) and height. The introduction of height represented a new feature, since the NAM had no height dependence and claimed to provide the aerosol concentration at deck height. However, when the ANAM development started, it was realized that the large particles that make up the $4^{\text {th }}$ mode are heavy and thus disperse inefficiently in the vertical. Since this could result in appreciable concentration gradients in the first 10-30 meters above the surface, height was included as a parameter in the regression analysis and was found to be of relevance. Since the initial analysis, ${ }^{27}$ the ANAM has been tuned to a more extensive dataset of Rotorod data acquired over a period of 15 years at the MPN platform in the North Sea, the North Atlantic and near Oahu HI. This last dataset resulted from the RED 2001 experiment and proved especially useful.

The improvement of ANAM4 over the original NAM can be judged from a performance factor, defined as the factor to within which the model predicts the observed aerosol concentrations $(\mathrm{r}>5 \mu \mathrm{m})$ at and below deck height. The ANAM4 yields a performance of approximately 3 , as compared to approximately $10-15$ for NAM. ${ }^{28}$ It should be noted that these performances have also been obtained for other Rotorod data sets than the ones used in the development of the ANAM4. The ANAM4 suggests that there is a considerable increase in aerosol extinction (and hence a reduction of transmission) due to the inclusion of larger particles and height dependence. Compared to NAM, ANAM predicts 10\% less transmission over a $15 \mathrm{~km}$ path at deck height, and up to $50 \%$ less transmission at 2 meters above the waves $(10 \mathrm{~m} / \mathrm{s}$ wind speed, long-wave infrared band). A recent contribution ${ }^{29}$ showed that this may have serious consequences for the performance of electro-optical surveillance sensors.

\section{THE PRODUCTION MODE}

The ANAM4 describes the freshly produced marine aerosols by two lognormals, i.e., the original $3^{\text {rd }}$ NAM mode centred around $2 \mu \mathrm{m}$ (nominal radius) and the $4^{\text {th }}$ mode centred around $8 \mu \mathrm{m}$. The two modes have different dependencies on wind speed and height. Intuitively, this seems not an optimal way to describe marine aerosol production. Thus, an effort was started to replace the $3^{\text {rd }}$ and $4^{\text {th }}$ modes by a single production mode. This task would respect the original NAM philosophy, which aims at providing a quick and reasonable estimate of aerosol extinction on the basis of simple (meteorological) input data by using empirically tuned relations. But at the same time, the ANAM could respond to some of the criticism formulated about NAM by taking into account some of the recent advances in our understanding of marine aerosol production and initial processes in the atmospheric surface layer. In particular, it was decided to replace the lognormal mode by a mode reflecting the shape of sea spray source function (S3F), the height dependence by an algorithm reflecting turbulent dispersion counterbalanced by gravity, and the humidity dependence by an explicit algorithm calculating the equilibrium radius of an aerosol particle in the atmosphere.

The first step consisted of a review ${ }^{5}$ of published equations for the S3F. As is well-known, the various estimates of the sea spray source function (both intensity and shape) vary considerably. While 10 years ago these estimates varied by more than 7 orders of magnitude, ${ }^{30}$ a recent workshop on the S3F agreed that the uncertainty is now reduced to about 1-2 orders of magnitude. There seems to be a consensus in the field that no single published source function describes correctly the sea spray behaviour over the complete radius range. One of the reasons for this is that source functions are generally based on experimental data, and that the equipment to provide this data is only capable of measuring a limited size range. Following recommendations by Schultz et al., ${ }^{31}$ it was decided that a combination of two source functions (for "small" and for "large" particles) would adequately cover the radius range of marine aerosol production.

For the larger particles, an algorithm by Smith and Harrison ${ }^{32}$ is suggested. Smith and Harrison base their source function on data accumulated during a cruise in the eastern North Atlantic, where they encountered moderate and high wind speeds up to $20 \mathrm{~m} / \mathrm{s}$. Their function is given as a flux function $\mathrm{dF} / \mathrm{dr}$ in $\left[\mu \mathrm{m}^{-1} \mathrm{~s}^{-1} \mathrm{~m}^{-2}\right]$ as a sum of two lognormals: 


$$
\frac{d F}{d r_{80}}=\sum_{i=1}^{2} A_{i} \exp \left\{-f_{i}\left[\ln \left(\frac{r}{r_{0 i}}\right)\right]^{2}\right\}
$$

Where $\mathrm{r}_{80}$ is the equilibrium radius at $80 \%$ humidity, $\mathrm{r}_{01}=3 \mu \mathrm{m}, \mathrm{r}_{02}=30 \mu \mathrm{m}, \mathrm{f}_{1}=1.5$ and $\mathrm{f}_{2}=1$, and the amplitudes are given by as function of $U_{10}$, i.e., the wind speed at 10 meters height:

$$
\left\{\begin{array}{l}
A_{1}=0.2 U_{10}^{3.5} \\
A_{2}=0.0068 U_{10}^{3}
\end{array}\right.
$$

For the smaller particles, the S3F given by Vignati et al. ${ }^{33}$ is suggested (the corrected equations given in ref. 31 have been used). This source function is based on shipboard measurements in the North Atlantic ${ }^{34}$ and consists of 3 lognormals. The S3F is a function of the instantaneous wind speed $U$ :

$$
\frac{d F\left(\log r_{80}\right)}{d \log r}=\sum_{i=1}^{3} \frac{N_{i}}{\sqrt{2 \pi} \log \sigma_{i}} \exp \left[-\frac{\left(\log r_{80}-\log R_{i}\right)^{2}}{2 \log ^{2} \sigma_{i}}\right]
$$

With parameters shown in the table below, and $d \log \mathrm{r}=0.1$.

Table 1: parameters for the Vignati S3F

\begin{tabular}{|l|l|l|l|}
\hline $\mathrm{i}$ & $\mathrm{N}_{\mathrm{i}}\left[\mathrm{cm}^{-2} \mathrm{~s}^{-1}\right]$ & $\mathrm{R}_{\mathrm{i}}[\mu \mathrm{m}]$ & $\sigma$ \\
\hline 1 & $10^{\wedge}(0.095 \mathrm{U}+0.283)$ & 0.2 & 1.9 \\
\hline 2 & $10^{\wedge}(0.0422 \mathrm{U}-0.288)$ & 2.0 & 2.0 \\
\hline 3 & $10^{\wedge}(0.069 \mathrm{U}-3.5)$ & 12.0 & 3.0 \\
\hline
\end{tabular}

It is suggested ${ }^{31}$ to switch between the two source functions at a dry radius of 4 microns. The conversion from radius at ambient humidity to dry radius ${ }^{35}$ is made on the assumption that the droplets only consist of water and salt $(\mathrm{NaCl})$ and that the salinity of the ocean amounts to 0.034 .

Thus, to evaluate the production mode in ANAM, a loop is made over all radii between (roughly) 0.01 and 100 microns. For each radius, the corresponding dry radius is calculated, which is subsequently used to invoke either the Vignati or the Smith \& Harrison source function. This procedure then yields the production flux $\mathrm{dF} / \mathrm{dr}$ in $\left[\mu \mathrm{m}^{-1} \mathrm{~s}^{-1} \mathrm{~m}^{-2}\right]$. Unfortunately, the two source functions do not match perfectly at the switch point of 4 microns dry radius. This is not just due to a difference in intensity, but also due to a difference in wind speed dependence. As a practical solution, it was decided to take one source function as "reference" and multiply the flux of the other source function by a radius-independent constant to match the reference source function. It was decided to use the Smith \& Harrison function as a reference, because their source function addresses the large particles. These particles have relatively short residence times and their concentration is thus likely to respond more directly to changes in wind speed.

In the next step, the production flux $\mathrm{dF} / \mathrm{dr}$ at the surface must be converted to a concentration at a particular height. One of the possibilities for this conversion consists of introducing a transfer function $\xi(\mathrm{z})$ :

$$
\frac{d F}{d r}\left[\mu m^{-1} s^{-1} m^{-2}\right]=\xi(z)\left[s m^{-1}\right] \cdot \frac{d N}{d r}(z)\left[\mu m^{-1} m^{-3}\right]
$$

The transfer function can be found by solving the dynamic (and thermodynamic) equations for the aerosols, including terms for macroscopic fluxes, turbulent diffusion, inertia and gravitational settling. ${ }^{10}$ The transfer function then applies to a stationary solution. This modeling approach and the shape of the final transfer function has been debated in many papers and will not be discussed here in detail. In many cases, equilibrium of fluxes is assumed resulting in a function 
$\xi(\mathrm{z})$ that decreases exponentially with height, ${ }^{36}$ although it has been demonstrated ${ }^{10}$ that this is an oversimplification for larger particles. For practical purposes, the transfer function has been implemented in ANAM as a 2-step process:

$$
\left\{\begin{array}{c}
\frac{d N}{d r}(z=0)=10^{P_{1}} \cdot \frac{d F}{d r} \\
\frac{d N}{d r}(z)=f(z) \cdot \frac{d N}{d r}(z=0)
\end{array}\right.
$$

Where $\mathrm{P}_{1}$ is an empirical tuning parameter, and $f(z)$ is a function that governs the decrease in concentration as function of height. An exponential shape was selected for $f(z)$ regardless of particle size, thereby neglecting near-surface effects due to wave-atmosphere interactions and the deviations from exponential behavior observed in numerical experiments. ${ }^{10}$ Since diffusion is less efficient for larger particles, the concentration gradient will be stronger for larger particles. In contrast, increased turbulent diffusion will lead to weaker gradients as wind speed increases. Thus, the basic form of $f(z)$ becomes:

$$
f(z)=\exp \left(-1.0 \cdot P_{2} \cdot f_{a}(r) \cdot f_{b}(U) \cdot z\right)
$$

Where $\mathrm{P}_{2}$ is an empirical tuning parameter and

$$
\begin{cases}f_{a}(r)={ }^{10} \log ^{P}{ }^{4} r_{80} & \left(r_{80} \geq 1.0 \mu m\right) \\ f_{b}(U)=3.0-\left(2 \cdot{ }^{10} \log U_{10}\right) & \left(U_{10} \geq 1 \mathrm{~m} / \mathrm{s}\right)\end{cases}
$$

And $\mathrm{P}_{4}$ is another empirical tuning parameter. For typical values of radii and wind speeds, and with values of $\mathrm{P}_{2}$ and $\mathrm{P}_{4}$ around unity, the value of $f(z)$ ranges between 0.02 and 0.08 . This corresponds to the vertical concentration gradients observed in the Rotorod dataset. ${ }^{7,10}$ Equation 7 implies that particles of radius less than $1 \mu \mathrm{m}$ have no vertical concentration gradient. These particles diffuse efficiently and yield a transfer function (stationary solution) that is almost independent of height. This choice may be justified in the domain of application of the ANAM, i.e., the lower 10-30 meters above the surface, but is subject to review at a later stage in this project.

Figure 1c shows the ANAM with the new production mode. Note that the new mode does not have the "traditional" lognormal shape and that it extends to very small (and large) radii. Since the ANAM is an empirical model, the next step in the introduction of the new production mode consisted of optimizing the tuning parameters to experimental data (see also next section). The initial tuning of the new production mode was done with two degrees of freedom, i.e., only tuning parameters $\mathrm{P}_{1}$ and $\mathrm{P}_{2}$ could be varied and $\mathrm{P}_{4}$ was fixed at a value of 2.0. Initial tuning revealed that the radius dependence of function $\mathrm{f}_{\mathrm{a}}(\mathrm{r})$ (equation 7) was not proper to correctly reproduce the experimental data. Therefore, parameter $\mathrm{P}_{4}$ was made available as an additional degree of freedom. Furthermore, the behavior of the Smith \& Harrison source function for very large particles did not properly match the experimental data. In view of the relatively large uncertainties in both the source function and the experimental data, it was not straightforward to select either one of these as the 'truth'. Thus, an additional tuning parameter $\mathrm{P}_{3}$ was introduced in the ANAM to modify the large particle behavior of the Smith \& Harrison source function above 20 microns radius:

$$
\left\{\begin{array}{l}
X=1.0-\frac{{ }^{10} \log 20.0}{{ }^{10} \log r_{80}} \\
Y=\max \left(0.25,1.0-X \cdot P_{3}\right)
\end{array}\right.
$$


Below 20 microns radius, $\mathrm{Y}$ is set to unity. The new factor $\mathrm{Y}$ is then used to (indirectly) modify the Smith \& Harrison source function as follows:

$$
\frac{d N}{d r}(z=0)=10^{P_{1} \cdot Y} \frac{d F}{d r}
$$

Obviously, it is hoped that the optimum value of $\mathrm{P}_{3}$ does not lead to substantial deviations of the shape of the Smith and Harrison source function.

\section{EMPIRICAL TUNING}

The tuning process consists of optimizing the values of P1 to P4 in such a manner that the ANAM reproduces best the available experimental data. Figure 1c shows that it is not straightforward to tune the new production mode, since this new mode overlaps the other modes (especially the $2^{\text {nd }}$ mode). Since the amplitude of these other modes also varies with the meteorological scenario, these modes will interfere with the tuning process. Therefore, it was decided to minimize interference by (initially) tuning the new production mode to particles with radii above 5 microns, i.e., the Rotorod database. ${ }^{21-26}$

The Rotorod technique is an active impactor sampling method, featuring silicon coated stainless steel rods that are mounted on a stabilized constant speed motor. The rods are spun to collect particles that leave an imprint in the silicon spray on the rods, which can be subsequently analyzed under a microscope. An important feature of the technique is that the size of the craters on the rods corresponds (closely) to the diameter of the particle at the time of impaction. ${ }^{7}$ The thus counted particles are distributed over bins (typically 10 between 10 and $100 \mu \mathrm{m}$ ) to yield an aerosol size distribution. A more detailed description can be found elsewhere, ${ }^{27}$ which also shows that the Rotorod technique reliably measures concentrations of particles with a radius larger than $5 \mu \mathrm{m}$. The upper radius limit is determined by statistics and ranges from 40 to $150 \mu \mathrm{m}$ depending on the concentration (basically determined by wind speed for sea-salt particles)

The Rotorod technique has been deployed at various campaigns in different regions of the world, ${ }^{37}$ but mostly at the MeetPost Noordwijk (MPN), a research tower located in the North Sea at $10 \mathrm{~km}$ off the Dutch coast. From these data, a relatively large composite MPN data set of 2078 observations (aerosol size distributions) can be formed, that should provide a good basis for a statistical analysis. The selection of MPN data implies that a bias could be introduced towards coastal conditions, even if only data acquired in long-fetch conditions are included in the analysis. Therefore, it is important to complete the analysis with open-ocean data, available from two experiments: the Cumulus trial in 1983 (117 observations), ${ }^{7}$ and the more recent RED experiment (297 observations). The open-ocean data was not mixed with the composite MPN-set, but treated as two separate data sets. Finally, a separate coastal dataset obtained at the FPN-platform in the German Bight (265 observations) was analyzed.

The tuning process of the new ANAM production mode was performed on the trial-and-error based used earlier in the ANAM4 development. ${ }^{28}$ In this procedure, the values of P1 - P4 were varied to obtain the best possible match between the aerosol concentrations as predicted by ANAM and as measured with the Rotorod impactors. The performance of a particular parameterization $P_{k}$ was calculated from a scatter plot between the experimental and modeled aerosol concentrations. In such a scatter plot, the standard deviation $\sigma$ of the data with respect to the line $\mathrm{y}=\mathrm{x}$ indicates the performance: the smaller the value of $\sigma$, the closer experimental and modeled concentrations. Furthermore, a performance factor ${ }^{38} F$ can be introduced $\left(F=10^{\sigma}\right)$ that is a measure how well the model predicts the experimental concentration: the prediction differs from the experimental concentration by a factor of $F$ or less in $67 \%$ of the cases.

Table 2: Tuning parameters and performance factors

\begin{tabular}{|l|l|l|l|l|l|l|l|c|}
\hline Dataset & $\mathrm{P} 1$ & $\mathrm{P} 2$ & $\mathrm{P} 3$ & $\mathrm{P} 4$ & & $\mathrm{~F}_{\text {ave }}$ ANAM5 & $\mathrm{F}_{\text {ave }}$ ANAM4 & $\mathrm{F}_{\text {ave }}$ NAM \\
\hline RED & 2.5 & 0.3 & 3.0 & 0.8 & & 2.2 & 2.6 & 20.0 \\
\hline Cumulus & 1.1 & 0.4 & 7.0 & 0.9 & & 3.6 & 7.8 & 8.7 \\
\hline MPN & 1.6 & 0.0 & 2.6 & 0.5 & & 6.1 & 3.3 & 8.8 \\
\hline FPN & 2.1 & 0.0 & 3.6 & 0.8 & & 3.9 & 2.8 & 16.4 \\
\hline
\end{tabular}


Table 2 presents the optimum values for P1 - P4 for the individual datasets, as well as the average performance factor $F_{\text {ave }}$. The average performance factor is calculated from the individual factors $\mathrm{F}$ for each of the Rotorod diameters (size bins) using a weighting procedure, in which less weight is given to the largest diameters to account for the reduced accuracy of the sampling device at these size ranges. The table also shows the average performance factors for the NAM and ANAM4 (individual $3^{\text {rd }}$ and $4^{\text {th }}$ modes).

Table 2 shows that the optimum values of P1 differ markedly from dataset to dataset. Since P1 controls the aerosol concentration at the surface, the results suggest that the concentration of large particles can vary considerably from experiment to experiment. This had been noted before ${ }^{28}$ and can only partially be explained by assuming that more aerosol particles should be present over a fully developed oceanic wave field than in a limited fetch condition at the North Sea. The possibility that flow distortion due to the ship or platform had an adverse impact on the Rotorod measurements cannot be excluded, which makes it quite difficult to decide on the 'best' value for P1.

Table 2 also shows that the optimum value for P2 equals zero for the coastal datasets, which indicates the total absence of a vertical concentration gradient. Some tests indicated that the performance factor could be further improved by using negative values of $\mathrm{P} 2$, which would indicate in higher concentrations aloft than at the surface. It is difficult to justify such a concentration profile, unless there is a rather strong advection component present that raises the concentrations aloft. In the coastal zone, such a component could be introduced by surf-generated aerosols that are advected out over sea. This possibility has not yet been further investigated.

The values for P3 and P4 show a reasonable consensus, with a notable exception for P3 / Cumulus. However, the performance factor for the Cumulus dataset is not very sensitive to the value of $\mathrm{P} 3$; $\mathrm{P} 3=3.5$ yields $\mathrm{F}_{\mathrm{ave}}=3.8$, which is close to the optimum value $\mathrm{F}_{\mathrm{ave}}=3.8$.

\section{DISCUSSION}

The Advanced Navy Aerosol Model has been reviewed as concerns its production mode. The two separate production modes $\left(3^{\text {rd }}\right.$ and $4^{\text {th }}$ modes in ANAM4) have been replaced by a single production mode in ANAM5. The shape of the new production mode is given by two sea spray source functions taken from literature, Vignati et al. ${ }^{33}$ and Smith and Harrison. ${ }^{32}$ The intensity of the new production mode in ANAM5 at a particular height above the surface is governed by a transfer function that depends on radius and wind speed. The production mode in ANAM5 has several tuning parameters that have been optimized by comparing ANAM5 concentration predictions to experimental aerosol data.

The performance of NAM, ANAM4 $\left(3^{\text {rd }}\right.$ and $4^{\text {th }}$ mode) and ANAM5 (new production mode) is compared in table 2. It should be kept in mind that the ANAM5 numbers represent different values of P1-P4 for each of the datasets. The 'final' ANAM5 will have a single set of values for P1-P4, which is likely to result in a slightly reduced performance for each individual dataset. ${ }^{28}$ As noted earlier, ${ }^{28}$ NAM is inadequate to model the concentration of larger aerosols. The NAM performance has been added as a warning to the Modtran ${ }^{1}$ community that NAM underestimates the concentration and hence overestimates the transmission, especially in the $8-12 \mu \mathrm{m}$ band. $^{29}$

The new ANAM5 performs better than ANAM4 for the open ocean datasets, but performs less than ANAM4 for the coastal datasets. The improvement for open ocean corroborates well with the concept of the new production mode, which basically provides the stationary solution for the vertical dispersion of freshly produced aerosols generated by an open ocean source function. The improvement in the performance factors thus provides confidence in this approach. The reduced performance in coastal areas shows that the new concept cannot improve over an empirically tuned lognormal. When the results were inspected in some more detail, it was found that the reduced performance resulted mainly from the fits in the smaller size bins. In other words: ANAM5 does a reasonable job for the largest particles in the coastal zone, but starts deviating when the size decreases. This may indicate again that there is an advection component in the coastal data (even at particle sizes of 20 microns). Such an advection component cannot be modeled by the new production mode, but should be introduced using the $2^{\text {nd }}$ (aged marine aerosol) mode of the ANAM. It is thus important to explore the presence of such an advection mode, because it will provide guidance for the selection of the final values of P1-P4.

As mentioned in the introduction, the review of the ANAM is not yet finished. The next step will be to review the advection or $2^{\text {nd }}$ mode of the (A)NAM. The mode shape and mode parameters will be reviewed and the mode will be 
adapted for the coastal zone. The amplitude of the $2^{\text {nd }}$ mode currently depends on the $24 \mathrm{~h}$ history of the wind speed reflecting production upwind and subsequent advection. In the coastal zone with limited fetch conditions, this concept breaks down. Once the $2^{\text {nd }}$ mode has been reviewed, tuning of the new ANAM5 can continue, using experimental data for smaller particles (just down to the sizes where the $0^{\text {th }}$ and $1^{\text {st }}$ modes start to interfere). It is hoped that this continued tuning also provides the final values for P1-P4 of the new production mode.

\section{ACKNOWLEDGEMENTS}

This work has been jointly sponsored by grants from the Joint Technology Office (contract FA9451-06-D-0008 / 0001) and the Office of Naval Research (ontract N68171-05-P-0147, Dr Ron Ferek). The authors acknowledge the instrumental efforts of Gerrit de Leeuw in obtaining the Rotorod database that has been used for the tuning of the new production mode. The enthusiastic participation of Gerard Kunz, Marcel Moerman and Leo Cohen in the Rotorod measurements is gratefully acknowledged. The authors also acknowledge the stimulating discussions with Gerrit de Leeuw in the initial selection process of the aerosol sea spray source functions for the new ANAM production mode. Dimitri Tsintikidis is acknowledged for optimizing the software used in the tuning process.

\section{REFERENCES}

1. Kneizys, F.X., L.W. Abreu, G.P. Anderson, J.H. Chetwynd, E.P. Shettle, A. Berk, L.S. Bernstein, D.C. Robertson, P. Acharya, L.S. Rothman, J.E.A. Selby, W.O. Gallery and S.A. Clough, The Modtran 2/3 report and Lowtran 7 model, Philips Laboratory PL/GPOS, Hanscom AFB, MA, 1996.

2. Mie, G. (1908). A contribution to the optics of turbid media, especially colloidal metallic suspensions. Ann. Phys. 25, 377-445, 1908 (In German).

3. Gathman, S.G., Optical properties of the marine aerosol as predicted by the Navy Aerosol Model, Opt. Eng. 22, 5762, 1983.

4. 5. Gathman, S.G. and K.L. Davidson, (1993) “The Navy Oceanic Vertical Aerosol Model”, TR1634 (Dec 1993), NCCOSC RDT\&E DIV, San Diego, CA 92152-5001.

5. Lewis, E.R. and S.E. Schwartz, Sea salt aerosol production: Mechanisms, methods, measurements and models - a critical review. AGU Geophysical Monograph 2004, ISBN 087590-417-3, 2004.

6. Van Eijk, A.M.J. and G. de Leeuw, Modeling aerosol extinction in a coastal environment. SPIE Proceedings 1688, 28-36, 1992.

7. DeLeeuw, G., Vertical profiles of giant particles close above the sea surface. Tellus 38B, 51-61, 1986.

8. DeLeeuw, G., K.L. Davidson, S.G. Gathman and R.V. Noonkester, Modeling of aerosols in the marine mixed-layer. SPIE Proceedings 1115, 27-1 to 27-8, 1989.

9. P. Berger (MIT-LL) and S. H. Hammel (SSC-SD), personal communication.

10. Van Eijk, A.M.J., B.J.S. Tranchant and P.G. Mestayer, SeaCluse : numerical simulation of evaporating sea spray droplets, J. Geophys. Res. 106, 2573-2588, 2001.

11. Fitzgerald, J.W., W.A. Hoppel and F. Gelbard (1998). A one-dimensional sectional model to simulate multicomponent aerosol dynamics in the marine boundary layer. I. Model description, J. Geophys. Res. D103, 16085-16102.

12. Hughes, H.G., Evaluation of the LOWTRAN6 Navy maritime aerosol model using 8 to $12 \mu \mathrm{m}$ sky radiances. Opt. Eng. 26, 1155-1160, 1987.

13. Gerber, H.E., Relative humidity parameterization of the Navy Aerosol Model (NAM), NRL report 8956, NRL Washington DC, 1985.

14. Gathman, S.G., A preliminary description of NOVAM, the Navy Oceanic Vertical Aerosol Model, NRL report 9200, NRL Washinton DC, 1989.

15. Koepke, P., M. Hess, I shult and E.P. Shettle. Global Aerosol Data Set. Report 243 of the Max Planck Institute for meteorology, Hamburg, Germany, ISBN 0937-1060, 1997.

16. Piazzola, J., A.M.J. van Eijk and G. DeLeeuw, An extension of the Navy Aerosol Model to coastal areas. Opt.Eng. 39, 1620-1631, 2000.

17. Piazzola, J., F. Bouchara, G. DeLeeuw and A.M.J. van Eijk, Development of the Mediterranean extinction code (MEDEX). Optical Engineering, 42, 912-924, 2003.

18. Houghton, H.G. and W.H. Radford, On the measurements of drop-size and liquid water content in fogs and clouds. Papers in Phys. Ocean and Meteorol. 6, Cambridge, MA, USA, 1938. 
19. Bricard, J., Etude de la constitution des nuages au sommet du Puy-de-Dôme. La Météorol. 15, 83-92, 1939.

20. Bricard, J., Contribution à l'étude des brouillards naturels. Annales de Phys. 14, 148-236, 1940.

21. DeLeeuw, G., Size distributions of giant aerosol particles close above sea level. J. Aerosol Sci. 17, 293-296, 1986.

22. DeLeeuw, G., Near-surface particle size distribution profiles over the North Sea. J. Geophys. Res. 92, 14631-14635, 1987.

23. DeLeeuw, G., Investigations on turbulent fluctuations of particle concentrations and relative humidity in the Marine Atmospheric Surface Layer. J. Geophys. Res. 94, 3261-3269, 1989.

24. DeLeeuw, G., Profiling of aerosol concentrations, particle size distributions and relative humidity in the atmospheric surface layer over the North Sea. Tellus 42B, 342-254, 1990.

25. Van Eijk, A.M.J. and L.H. Cohen, TNO-FEL Rotorod aerosol samplers. TNO Physics and Electronics Laboratory, report FEL-97-I248, 1997.

26. Cohen, L.H. and A.M.J. van Eijk, Rotorod aerosol samplers 2. TNO Physics and Electronics Laboratory, report FEL-98-I338, 1998.

27. Gathman, S.G., A.M.J. van Eijk and L.H. Cohen, Characterizing large aerosols in the lowest levels of the marine atmosphere, SPIE Proceedings 3433, 41-52, 1998.

28. Van Eijk, A.M.J., L.H. Cohen, L.J. Navarro and G. DeLeeuw, Near-surface aerosol transmission in the marine environment, SPIE Proceedings 4884, 160-169, 2002.

29. Hammel, S.H., D. Tsintikidis and A.M.J.Van Eijk, ANAM vs NAM: is the difference significant?, SPIE Proceedings 5891, 2005.

30. Andreas, E.L., A new sea spray generation function for wind speeds up to $32 \mathrm{~m} / \mathrm{s}$, J. Phys. Oceanogr., 28, 2175 2184, 1998.

31. Schultz, M., G. DeLeeuw and Y. Baltanski, Sea-salt aerosol source functions and emissions. In: Emission of atmospheric trace compounds, eds: C. Granier, P. Artaxo, C.E. Reeves, Ed. Kluwer, 347-372, 2003.

32. Smith, M.H. and N.M. Harrison, The sea spray generation function, J. Aerosol. Sci., 29, S189-S190, 1998.

33. Vignati, E., G. DeLeeuw and R. Berkowicz, Modelling coastal aerosol transport and effects of surf-predicted aerosols on processes in the marine atmospheric boundary layer, J. Geophys. Res., 106, 20225-20238, 2001.

34. O’Dowd, C.D.,M.H. Smith, I.E. Consterdine and J.A. Lowe, Marine aerosol, sea-salt and the marine sulphur cycle: a short review, Atmos. Environ., 31, 73-80, 1997.

35. Andreas, E.L., Thermal and size evolution of sea spray droplets. CRREL report 89-11, US Army cold regions research and engineering laboratory, Hanover NH, 37p, NTIS: AD-A210484.

36. Toba, Y., On the giant sea-salt particles in the Atmosphere. II. Theory of the vertical distribution in the 10-m layer over the ocean, Tellus 17, 365-382, 1965.

37. S.G. Gathman and A.M.J. van Eijk, "Electro optical propagation just above wave tops as predicted by ANAM, the advanced Navy aerosol model", AGARD Sensor Propagation panel specialist's meeting on EO propagation, Signature and System Performance under adverse meteorological conditions, considering out-of-area operations, 16-19 March 1998, Naples, Italy.

38. A.M.J. van Eijk and G. DeLeeuw, "Modeling aerosol extinction in a coastal environment", SPIE Proceedings 1688, 28-36, 1992. 